\title{
Infections à papillomavirus
}




\section{Springer}

Paris

Berlin

Heidelberg

New York

Hong Kong

Londres

Milan

Tokyo 
Joseph Monsonego

\section{Infections à papillomavirus}

État des connaissances,

pratiques et prévention vaccinale

Springer 


\title{
Joseph Monsonego
}

174, rue de Courcelles

75017 Paris

ISBN-10 : 2-287-33479-3 Springer Paris Berlin Heidelberg New York ISBN-13 : 978-2-287-33479-5 Springer Paris Berlin Heidelberg New York

\author{
(C) Springer-Verlag France, Paris 2006 \\ Imprimé en France
}

\section{Springer-Verlag France est membre du groupe Springer Science + Business Media}

Cet ouvrage est soumis au copyright. Tous droits réservés, notamment la reproduction et la représentation, la traduction, la réimpression, l'exposé, la reproduction des illustrations et des tableaux, la transmission par voie d'enregistrement sonore ou visuel, la reproduction par microfilm ou tout autre moyen ainsi que la conservation des banques de données. La loi française sur le copyright du 9 septembre 1965 dans la version en vigueur n'autorise une reproduction intégrale ou partielle que dans certains cas et en principe moyennant le paiement des droits. Toute représentation, reproduction, contrefaçon ou conservation dans une banque de données par quelque procédé que ce soit est sanctionnée par la loi pénale sur le copyright.

L'utilisation dans cet ouvrage de désignations, dénominations commerciales, marques de fabrique, etc. même sans spécification ne signifie pas que ces termes soient libres de la législation sur les marques de fabrique et la protection des marques et qu'ils puissent être utilisés par chacun.

La maison d'édition décline toute responsabilité quant à l'exactitude des indications de dosage et des modes d'emplois. Dans chaque cas il incombe à l'usager de vérifier les informations données par comparaison à la littérature existante.

SPIN : 11739364

Maquette de couverture: Jean-François Montmarché 


\section{Avant-propos}

Avec près de 500000 cas annuels et 230000 décès, le cancer du col est la deuxième cause de cancer chez la femme dans le monde. En Europe, où beaucoup de pays ont mis en place un programme de dépistage du cancer du col, la maladie se situe au troisième rang des cancers féminins en termes d'incidence. En France en 2000, le cancer du col affecte 3400 femmes et occasionne 1000 décès chaque année. Le risque de développer un cancer du col est estimé à $1 \%$ dans les pays développés et à $5 \%$ dans les pays en voie de développement.

Le triage des frottis ASC-US, qui représentent 2,5 à $4 \%$ de la totalité des frottis, basé sur le test HPV, est largement admis, recommandé et remboursé en France. Après l'âge de 30 ans, le groupe des femmes à frottis négatif - HPV à haut risque positif représente environ 5 à $7 \%$ de la population. Parmi ces femmes, il est possible désormais d'identifier avec plus de précision celles qui sont à risque réel de lésions précancéreuses sous-jacentes en utilisant des marqueurs moléculaires performants. Il est prouvé que la persistance virale ainsi que la charge virale élevée sont des indicateurs fiables de lésions précancéreuses. Plus récemment, le génotypage viral permet de répondre de manière plus précise à cette question. En effet, il est démontré que les papillomavirus 16 et 18 sont les types viraux auxquels les femmes sont le plus souvent exposées dans leur jeune âge, en particulier en Europe et en Amérique du Nord, et que ce sont sur ces types viraux que la persistance, après l'âge de 30 ans, est le plus souvent observée. Ainsi la persistance à HPV 16 ou 18 est un marqueur lésionnel encore plus fiable que la persistance dans un cocktail de papillomavirus à risque. D’autres marqueurs des lésions de haut grade sont aujourd'hui disponibles, les ARN messagers des gènes $\mathrm{E} 6-\mathrm{E} 7$ des papillomavirus à risque sont le plus souvent exprimés dans ces lésions. La détection d'ARN messagers E6E7 de certains papillomavirus à risque a une grande probabilité d'être associée à une lésion précancéreuse. Le marquage des cellules par la protéine P16ink4a en cytologie ou en histologie peut aider le pathologiste à reconnaître un sujet à risque lors du dépistage ou à améliorer l'assurance de qualité en histologie, en particulier pour les CIN 1. 
Malgré le succès considérable enregistré par le dépistage cytologique pour prévenir le cancer du col, le frottis n'a pas bénéficié de tous les espoirs auxquels on pouvait s'attendre pour réduire à une large échelle son incidence. Dans beaucoup de pays, le frottis de dépistage a transformé le cancer du col d'une maladie mortelle en une pathologie rare. Par ailleurs, le dépistage ne semble profiter qu'à une infime partie de la population mondiale alors qu'une large proportion de ceux qui en bénéficient endure ses faiblesses.

L'impact de l'infection à papillomavirus dans la population est considérable. Plus d'une femme sur deux a été exposée aux HPV durant sa vie, $10 \%$ en sont porteuses de façon chronique et parmi elles une femme sur cinq risque de développer un cancer du col en l'absence de dépistage ou en cas de dépistage défaillant.

Le fait que le cancer du col soit la conséquence ultime de l'infection chronique à papillomavirus procure l'extraordinaire opportunité de prévenir la maladie par la vaccination. Les HPV 16 et 18 sont responsables de $70 \%$ des cancers du col dans le monde. Ces types sont reconnus comme les principaux types viraux associés au risque de développer des lésions précancéreuses et cancéreuses tant chez les femmes à frottis normal que chez celles ayant des anomalies cytologiques de type ASC-US ou bas grade.

Un vaccin prophylactique, pour prévenir les lésions précancéreuses et cancéreuses, composé de ces types viraux, devrait sauver des vies, réduire l'anxiété, les interventions coûteuses et aurait un bénéfice individuel et collectif non négligeable.

Les vaccins HPV prophylactiques verront le jour avant la fin de l'année 2006. Basés sur la production de particules virales non infectantes issues de la protéine majeure de capside L1 qui a la particularité de s'autoassembler spontanément sous la forme de pseudo-particules virales (VLP L1), les essais cliniques qui bénéficient de plus de quatre ans de suivi postvaccinal montrent que les vaccins prophylactiques 16 et 18 induisent une forte production d'anticorps neutralisants avec un minimum d'effets secondaires, une protection de $100 \%$ de l'infection à HPV 16 et 18 persistante et des CIN de haut grade associées. Les résultats issus des essais cliniques utilisant le vaccin bivalent $\left(\right.$ Cervarix $^{\odot}$ ) de GSK et le vaccin quadrivalent $\left(\right.$ Gardasil $^{\circ}$ ) de Merck sont concordants dans ce sens bien que chacun d'entre eux ait ses particularités propres. La vaccination HPV réduirait de moitié la fréquence des frottis anormaux, le nombre de colposcopies et biopsies dirigées ainsi que les traitements des lésions précancéreuses. Associée au dépistage, on estime qu'une large couverture vaccinale induirait une réduction de l'incidence de ce cancer de $90 \%$. L'instauration d'une vaccination systématique pour les jeunes filles âgées de 11 à 16 ans, avec pendant 4 à 5 ans, un rattrapage progressif des cohortes de jeunes femmes âgées de 17 à 25 ans, correspond au positionnement démontré de la vaccination. L'introduction du vaccin HPV, couplé au dépistage, permettrait de démarrer l'âge de début de ce dépistage plus tard et, à moyen terme, d'autoriser sans risque majeur un espacement du dépistage à 3-5 ans. Les carences dans l'observance au dépistage constituent en effet l'un des échecs des campagnes de dépistage actuelles. Il faudra cependant veiller à ce qu'il n'y ait pas de relâchement conséquent de ce dépistage ou de la prévention des IST, étant donné les messages qui vont entourer une vaccination anti-cancer ou anti-IST. 
Cet ouvrage au contenu diversifié dresse un état des lieux des connaissances et des perspectives sur l'infection à papillomavirus et des pathologies associées. Il s'adresse aux acteurs principaux impliqués dans le dépistage, le diagnostic, le traitement et la prévention des précancers et cancers du col.

Dans cet environnement en pleine mutation, marqué par beaucoup d'évidences et quelques incertitudes, décrypter les nouveaux enjeux, anticiper les évolutions à court et moyen terme, rappeler les stratégies cohérentes de prise en charge et des pratiques est l'un des objectifs de cet ouvrage qui se veut avant tout informatif et didactique.

D'ores et déjà se dessinent les contours d'une nouvelle pratique clinique : un dépistage cyto-moléculaire ciblé et performant, à un rythme espacé, auprès d'une population potentiellement immunisée. L'éradication du cancer du col deviendra alors un objectif réaliste, à condition que des campagnes d'information et d'éducation adaptées génèrent une forte mobilisation des femmes et des professionnels de santé.

Joseph MONSONEGO

\section{Du même auteur}

Dysplasies du col et papillomavirus humains. Maloine Paris, 1988

New Developments in Cervical Cancer Screening and Prevention. Blackwell

Science, Oxford, 1997

Emerging Issues on HPV Infections: From Science to Practice. Karger Basel, 2006 


\section{Sommaire}

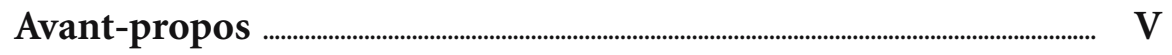

ÉTAT DES CONNAISSANCES …….......................................... 1

Infections génitales à HPV. Bases fondamentales ......................................... 3

Caractéristiques des HPV. Biologie virale ................................................................ 4

Données épidémiologiques.Transmission ............................................................. 8

Indicateurs pronostiques de l'infection à HPV à risque ............................ 16

Histoire naturelle de l'infection à HPV et des CIN ....................................... 23

Mécanisme de la carcinogenèse ......................................................................................... 25

Réponses immunitaires anti-HPV ............................................................................ 30

Conclusion. Différences fondamentales entre l'infection à HPV

à bas risque et à haut risque ..................................................................................................... 35

Cancer du col en France et dans le monde ......................................................... 47

Cancer du col utérin en France ............................................................................................... 48

Cancer du col utérin en Europe ......................................................................................... 53

Cancer du col utérin dans le monde ................................................................................. 58

DIAGNOSTIC DE L'INFECTION À PAPILLOMAVIRUS ET DES LÉSIONS ASSOCIÉES ……………............................................................ 65

Apport de la biologie. Le test HPV .................................................................... 67

Principales techniques ............................................................................................................ 68

Nouvelle génération de tests HPV .................................................................................. 73

Apport de la P16ink4a dans le dépistage et le diagnostic ....................... 78

Conlusion ...................................................................................................................................... 79 


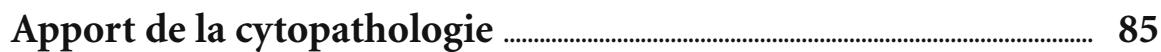

Recueil en milieu liquide .................................................................................................... 86

Classification de Bethesda .......................................................................................................... 93

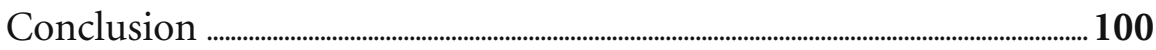

Apport de la colposcopie …......................................................................................... 103

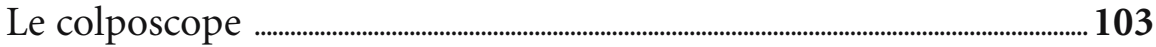

Trois temps de l'examen ..............................................................................................................104

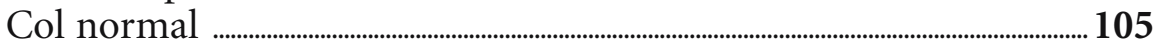

Images élémentaires ...........................................................................................................107

Situations particulières et modifications physiologiques .........................107

Procédures de diagnostic .........................................................................................................109

Impressions colposcopiques ........................................................................................111

Colposcopie satisfaisante et non satisfaisante ………............................................113

Reproductibilité de la colposcopie ..................................................................................113

Effets secondaires potentiels de la colposcopie ..................................................114

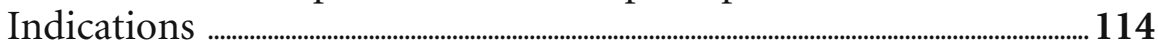

Terminologies ...................................................................................................................114

Compte rendu de colposcopie .............................................................................................117

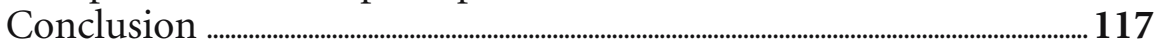

PRISE EN CHARGE DES LÉSIONS GÉNITALES À HPV ................... 119

Dépistage du cancer du col : le point actuel ...................................................... 121

Histoire des frottis des femmes qui développent un cancer

invasif du col. Problématique du dépistage ............................................................ 121

Limites du dépistage basé sur le frottis conventionnel .................................122

Optimisation de la sensibilité du dépistage .........................................................123

Test HPV et dépistage primaire : apport des études actuelles ............126

Le test HPV peut-il remplacer le frottis de dépistage ? ................................129

Apport des études économiques ..............................................................................130

Peut-on éviter les dérives ? Problèmes non encore résolus ...................130

Consensus et rapports disponibles ...........................................................................131

Les perspectives d'un vaccin HPV prophylactique vont-elles modifier le dépistage ? .................................................................................................132

Conclusion .......................................................................................................................... 133

Prise en charge d'un frottis anormal : apport de la colposcopie et du test HPV en pratique clinique

Apport de la colposcopie dans la prise en charge des frottis anormaux

Colposcopie et test HPV positif de dépistage 
Colposcopie et test HPV de seconde intention

Colposcopie pour le suivi des patientes avec atypies cytologiques mineures (ASC-US/LSIL), avec une CIN 1 non traitée ou après traitement d'une CIN

Patientes présentant des condylomes acuminés génitaux externes ou papulose bowénoïde (VIN 3)

Partenaire présentant des lésions à HPV

Populations à risque

Symptômes persistants à titre de leucorrhées et de métrorragies, en particulier métrorragies postcoïtales

Colposcopie dans les situations particulières

Colposcopie de dépistage

Conclusion

Traitement des lésions génitales à $\mathrm{HPV}$.

Indications, interprétation du test HPV et des marqueurs moléculaires

Comprendre et interpréter les résultats

Apports du test HPV en pratique clinique

Prise en charge après ASC-US/ASC-H

Prise en charge après frottis L-SIL

Frottis et test HPV en dépistage primaire

Sept points clés pour une bonne utilisation clinique

du test HPV

Six informations pour les patientes .................................................................................. 177

Méthodes de diagnostic

Lésions génitales externes à HPV

Bilan et prise en charge des condylomes acuminés génitaux

externes en pratique quotidienne

Néoplasies intravulvaires

VACCINATION PROPHYLACTIQUE

ANTI-PAPILLOMAVIRUS

État des connaissances et perspectives

pour la pratique clinique

Cancer du col utérin. Les chiffres

Rôle des papillomavirus (HPV) 
Vaccination HPV comme approche de prévention primaire du cancer du col 218

Conclusion

Espoir et promesses de la vaccination HPV

Annexe

Aspects colposcopiques des lésions HPV associées (planches en couleur) 